\title{
The prognostic and predictive value of osteopontin in colon adenocarcinoma
}

\author{
Yasemin Akca', Murat Alper ${ }^{2}$ \\ ${ }^{I}$ Department of Medical Pathology, Gaziantep University, Faculty of Medicine, Gaziantep, Turkey \\ ${ }^{2}$ Department of Medical Pathology, Diskapı Ylldırım Beyazıt Training and Research Hospital, Ankara, Turkey
}

\begin{abstract}
A BSTRACT
Aim: The identification of cellular pathways in colorectal tumor biology is essential for early diagnosis, treatment, and post-treatment follow-up. Osteopontin is an extracellular matrix protein that has regulatory physiological functions and roles in apoptosis, proliferation, adhesion, invasion, and tumor metastasis. In this study, we aimed to determine the prognostic and predictive value of osteopontin in colon adenocarcinoma. Our study investigated whether osteopontin expression had any prognostic or predictive use in colon adenocarcinoma and also if there were any differences between adenocarcinoma and adenoma.

Methods: Fifty of these colonic specimens were adenocarcinoma, 16 were adenomatous polyps, and 10 were nontumoral colonic tissue that served as a control group. We used a two-tiered evaluation system that examined both the staining intensity and the percentage of staining.

Results: The staining scores of tumors with vascular invasion were significantly higher than those of tumors without vascular invasion. In addition, the tumoral tissues' osteopontin staining scores were significantly higher than the score of polyps.

Conclusion: If future studies support our results, we suggest that osteopontin may be an important biomarker for predicting or detecting vascular invasion in tumors and could be useful in tumor-adenomatous polyp differentiation. Therefore, osteopontin can provide helpful information in the diagnosis and prognosis of colon adenocarcinoma.
\end{abstract}

Keywords: Osteopontin, colon adenocarcinoma, adenomatous polyp, vascular invasion.

$\triangle$ Dr. Yasemin Akca

Department of Medical Pathology, Gaziantep University,

Faculty of Medicine, Gaziantep, Turkey

E-mail: dryaseminakca@gmail.com

Received: 2020-10-04 Revised: 2020-12-29

Accepted: 2021-02-20 / Published online: 2021-04-01

\section{Introduction}

Colorectal carcinoma is the most common carcinoma of the gastrointestinal system and is also the leading cause of death from cancer in some countries [1]. Depending on the incidence, morbidity, and mortality rates, colon carcinoma has a very important place among all tumors. Cancer development is a complex process that involves combinations of various tumor and tumor stromal-derived growth factors and cytokines. As demonstrated in other types of cancer, the identification of cellular pathways in colorectal tumor biology creates new opportunities for early diagnosis and future treatments. For this reason, many studies are being carried out at the cellular and molecular levels, and molecular level findings in particular play an important role in the prognosis since they assist in predicting the clinical course, patient follow-up, and application of treatment procedures. Some 
markers are also used to assess the metastatic potential of a tumor [1-7].

Osteopontin was first isolated from bone tissue in 1979. It a structural protein of bone tissue and an extracellular matrix protein found in the phosphoglucoprotein structure; osteopontin is also detected in other tissues of the body [2]. This substance plays many roles in cell-matrix interactions, the modulation of cell functions, and carcinogenesis [3]. These cancers include (but are not limited to) malignant breast cancer, osteosarcoma, melanoma, ovarian cancer, endometrial cancer, cervical cancer, renal cancer, oral cancer, esophageal cancer, gastric cancer, colorectal cancer, pancreatic cancer, liver cancer, lung cancer, head and neck cancer, glioblastoma, skin cancer, thyroid cancer, and sarcoma [4-7].

In this study, we researched whether there was a significant relationship between osteopontin expression and prognostic parameters such as age, sex, localization, differentiation grade, lymph node involvement, vascular invasion, and cancer stage in colon adenocarcinomas. We also investigated whether there was any difference between adenocarcinoma and adenoma, which is an accepted precursor lesion of adenocarcinoma, for osteopontin expression.

\section{Materials and Methods}

We performed a retrospective review of 50 patients who underwent colon resection and were diagnosed with adenocarcinoma at our hospital and 16 patients with adenoma that were diagnosed by biopsy during colonoscopy. Ethics Committee approval was obtained as Decision Number 25/08. Ten nontumoral colon tissues were selected as the control group. Information and patient records were obtained from the hospital automation system. Hematoxylin and Eosin (H\&E) stained slides of archival paraffin blocks were evaluated. The degrees of differentiation were grouped as follows: well-differentiated if a tumor contained more than $95 \%$ of the glandular structure, moderately differentiated if the tumor involved $5=-95 \%$ of the glandular structure, and poorly differentiated if it contained $5-50 \%$ the glandular structure [1]. The tumor stage was assessed using TNM (Tumor-NodeMetastasis) classification. The cases were divided into two groups according to age $(<65$ or $\geq 65$ years), lateralization (right or left according to embryological development), and positivity (positive or negative) concerning lymph node involvement and the presence of vascular invasion. For each case, slides were selected to show the highest grade of a tumor and also examples of nontumoral tissue.

\section{Immunohistochemical staining method}

Immunohistochemistry was performed on the deparaffinized tissue sections obtained from formalin-fixed and paraffin-embedded tissue blocks. We used an automated slide stainer (Leica Bond Max device and osteopontin rabbit polyclonal antibody (RB-9097-R7) (Thermo Scientific) at a 1:50 dilution. The device staining procedure requires boiling the tissue sections in a 10-mM citrate buffer at a $\mathrm{pH}$ of 6:0 for 20 minutes, followed by cooling for 20 minutes.

\section{Scoring of the staining method}

Stomach adenocarcinoma was used as a positive control, and cytoplasmic staining with osteopontin was confirmed when slight nuclear staining was observed in cells where the staining was intense. We evaluated cytoplasmic staining in tumor epithelial cells. No significant specific staining pattern was observed in stromal structures. To assess the percentage of staining, we used a two-tiered scoring system 
that included a combination of the staining intensity and the Allred Scoring System [8]. We developed a grading scale as follows:

Staining intensity

Score of 0: None

Score of 1: Weak

Score of 2: Moderate

Score of 3: Strong

Percentage of staining

Score of 0: 0\%

Score of $1: \leq 5 \%$

Score of 2: 6-10\%

Score of 3: 11-33\%

Score of 4: 34-66\%

Score of 5: 67-100\%

The sum of these two scorings led to a final score:

0-1: Score of 0

2-3: Score of $1(+)$

4-6: Score of $2(++)$

7-8: Score of $3(+++)$

\section{Statistical analysis}

Our statistical analysis was performed using the Statistical Package for the Social Sciences software, version 20.0. Categorical variables were presented as frequencies and percentages. Descriptive statistics were shown as the mean + standard deviation (SD) for numerical cut-off variables, and categorical variables were the number of cases and percentages (\%). Pearson's Chi-Square, Fisher's Chi-square, or likelihood ratio tests were used to evaluate the categorical variables. Results with a $p<0.05$ were considered statistically significant.

\section{Results}

The research population consisted of 76 people, $42(55.3 \%)$ males and $34(44.7 \%)$ females. The youngest patient was aged 40 , the oldest was 80 years old, and $53 \%$ of the entire population were among the geriatric group (65 years and over). Tumors were found in $65.8 \%(\mathrm{n}=50)$ of the population, polyps were noted in $21.1 \%$ ( $\mathrm{n}$ $=16$ ), and nontumoral tissues were present in $13.2 \%(\mathrm{n}=10$; Table 1$)$.

There was low grade dysplasia in $56.3 \%(n=9)$ of the polyps and high grade dysplasia in $43.8 \%$ $(n=7)$. Of the tumors, $86 \%(n=43)$ were moderately differentiated, $8 \%(\mathrm{n}=4)$ were poorly differentiated and $6 \%(\mathrm{n}=3)$ were well differentiate. In $54 \%(n=27)$ of tumor cases. Lymph node metastasis was present, and $46 \%$ $(\mathrm{n}=23)$ presented with vascular invasion. According to TNM classification, 64\% $(n=32)$ of the cases had invasion into subserosa (T3), $12 \%(\mathrm{n}=6)$ into muscularis propria (T2), $22 \%$ $(\mathrm{n}=11)$ into serosa $(\mathrm{T} 4)$, and $2 \%(\mathrm{n}=1)$ into both mucosa and submucosa (T1). Gauging the tumors by the AJCC 2017 TNM stage, 36\% (n $=18)$ were stage IIIB, $34 \%(\mathrm{n}=17)$ were stage IIA, $12 \%(\mathrm{n}=6)$ reached stage IIIC, $10 \%(\mathrm{n}=$ 5) presented as stage I, $4 \%(n=2)$ were stage IIB, $2 \%(\mathrm{n}=1)$ were stage IIIA and $2 \%(\mathrm{n}=1)$ were stage IVA (Table 1).

In the geriatric age group, $51.4 \%(\mathrm{n}=18)$ had score 3 staining and $48.6 \%(n=17)$ had score 2 staining present. The under 65 age group had score 3 staining present in $74.2 \%(\mathrm{n}=23)$ and score 2 staining in $25.8 \%(n=8 ; p=0.059)$. Score 3 staining was also present in $81 \%(\mathrm{n}=$ 17) of right localized tumors but was found in only $65.5 \%(\mathrm{n}=19)$ of left localized tumor cases; these findings were not statistically significant $(p=0.341)$ (Table 2).

A significant relationship could not be found in epidemiological data comparisons, but significant differences were found between diagnoses and tumor characteristics, specifically with the staining score $(p<0.001)$. Accordingly, we found that the score 2 staining rate was higher in adenomatous polyps, and the score 3 staining rate was higher in tumors (Table 2). 
Score 3 staining was observed in all poorly differentiated tumors, in $70.3 \%(n=26)$ of moderately differentiated tumors and $66.7 \%$ ( $=2$ ) of well-differentiated tumors. An increase in the ratio of Score 3 staining corresponding with decreasing tumor differentiation did not show statistical significance $(p=0.637)$ (Table $2)$. Score 3 staining was significantly higher in tumors with vascular invasion than in tumors without it $(95.7 \% / 51.9 \%, p=0.001)$. While the staining score was not statistically significant for tumors with and without lymph node metastases, those with lymph node metastases had higher scores than those without (74.1\%/69.6\%, $p=0.726)$. No significant difference was found between tumor stage and subgroups $(p=0.801)$ or depth of invasion $(p=$ 0.296) (Table 2), (Figure 1).

Table 1. Demographic features and staining scores of the cases.

\begin{tabular}{|c|c|c|c|c|c|}
\hline Variables & $\begin{array}{l}\text { All population } \\
\qquad \begin{array}{l}(\mathrm{N}=76) \\
\mathrm{N}(\%)\end{array}\end{array}$ & $\begin{array}{c}\text { Tumors } \\
(\mathbf{N}=\mathbf{5 0}) \\
\mathbf{N}(\%)\end{array}$ & $\begin{array}{l}\text { Polyps } \\
(\mathbf{N}=16) \\
\text { N }(\%)\end{array}$ & $\begin{array}{c}\text { Nontumoral } \\
\text { Tissues } \\
(\mathbf{N}=10) \\
\text { N }(\%)\end{array}$ & $p$ \\
\hline Age $(\mathrm{Med} \pm \mathrm{SD})$ & $66.4 \pm 11.2$ & $66.1 \pm 11.7$ & $67.4 \pm 9.6$ & $0(0)$ & \\
\hline Unknown & $0(0)$ & $0(0)$ & $0(0)$ & $10(100)$ & \\
\hline$<65$ & $31(47)$ & $24(48)$ & $7(43.8)$ & $0(0)$ & \multirow{2}{*}{0.993} \\
\hline$\geq 65$ & $35(53)$ & $26(52)$ & $9(56.3)$ & $0(0)$ & \\
\hline \multicolumn{6}{|l|}{ Gender } \\
\hline Female & $34(44.7)$ & $25(50)$ & $6(37.5)$ & $3(30)$ & \multirow{2}{*}{0.404} \\
\hline Male & $42(55.3)$ & $25(50)$ & $10(62.5)$ & $7(70)$ & \\
\hline \multicolumn{6}{|l|}{ Diagnosis } \\
\hline Nontumoral colon tissue & $10(13.2)$ & $0(0)$ & $0(0)$ & $10(100)$ & \multirow{3}{*}{-} \\
\hline Adenocarcinoma & $50(65.8)$ & $50(100)$ & $0(0)$ & $0(0)$ & \\
\hline Adenomatous polyps & $16(21.1)$ & $0(0)$ & $16(100)$ & $0(0)$ & \\
\hline \multicolumn{6}{|l|}{ Staining intensity } \\
\hline Score 0 & $10(13.2)$ & $0(0)$ & $0(0)$ & $10(100)$ & \multirow{4}{*}{$<0.001 *$} \\
\hline Score1 & $17(22.4)$ & $9(18)$ & $8(50)$ & $0(0)$ & \\
\hline Score 2 & $32(42.1)$ & $28(56)$ & $4(25)$ & $0(0)$ & \\
\hline Score 3 & $17(22.4)$ & 13(26) & $4(25)$ & $0(0)$ & \\
\hline \multicolumn{6}{|l|}{ Percentage of staining } \\
\hline Score 0 & $10(13.2)$ & $0(0)$ & $0(0)$ & $10(100)$ & \multirow{4}{*}{$<0.001 *$} \\
\hline Score 3 & $5(6.6)$ & $3(6)$ & $2(12.5)$ & $0(0)$ & \\
\hline Score 4 & $16(21.1)$ & $7(14)$ & $9(56.3)$ & $0(0)$ & \\
\hline Score 5 & $45(59.2)$ & $40(80)$ & $5(31.3)$ & $0(0)$ & \\
\hline \multicolumn{6}{|l|}{ Final score } \\
\hline Score 0 & $10(13.2)$ & $0(0)$ & $0(0)$ & $10(100)$ & \multirow{3}{*}{$<0.001 *$} \\
\hline Score 2 & $25(32.9)$ & $14(28.0)$ & $11(68.8)$ & $0(0)$ & \\
\hline Score 3 & $41(53.9)$ & $36(72.0)$ & $5(31.2)$ & $0(0)$ & \\
\hline
\end{tabular}


Table 2. Distribution of final scores according to demographic and clinical findings.

\begin{tabular}{|c|c|c|c|c|}
\hline \multirow[b]{2}{*}{ Variables } & \multicolumn{3}{|c|}{ Final score } & \multirow[b]{2}{*}{$p$} \\
\hline & $\begin{array}{c}\text { Score 0 } \\
\mathbf{N}(\%) \\
\end{array}$ & $\begin{array}{c}\text { Score } 2 \\
\mathbf{N}(\%) \\
\end{array}$ & $\begin{array}{l}\text { Score } 3 \\
\mathbf{N}(\%) \\
\end{array}$ & \\
\hline \multicolumn{5}{|l|}{ Age } \\
\hline Under 65 & $0(0)$ & $8(25.8)$ & $23(74.2)$ & \multirow{2}{*}{0.059} \\
\hline 65 and over & $0(0)$ & $17(48.6)$ & $18(51.4)$ & \\
\hline \multicolumn{5}{|l|}{ Gender } \\
\hline Female & $3(8.8)$ & $11(32.4)$ & $20(58.8)$ & \multirow{2}{*}{0.649} \\
\hline Male & $7(16.7)$ & $14(33.3)$ & $21(50)$ & \\
\hline \multicolumn{5}{|l|}{ Diagnosis } \\
\hline Normal colon tissue & $10(100)$ & $0(0)$ & $0(0)$ & \multirow{6}{*}{$<0.001 *$} \\
\hline Adenocarcinoma & $0(0)$ & $12(27.3)$ & $32(72.7)$ & \\
\hline Mucinous Adenocarcinoma & $0(0)$ & $2(33.3)$ & $4(66.7)$ & \\
\hline Tubular Adenoma & $0(0)$ & $6(75)$ & $2(25)$ & \\
\hline Tubulovillous Adenoma & $0(0)$ & $2(50)$ & $2(50)$ & \\
\hline Villous Adenoma & $0(0)$ & $3(75)$ & $1(25)$ & \\
\hline Dysplasia degree in polyps & $0(0)$ & & & \\
\hline Low & $0(0)$ & $6(66.7)$ & $3(33.3)$ & \multirow{2}{*}{0.838} \\
\hline High & $0(0)$ & $5(71.4)$ & $2(28.6)$ & \\
\hline \multicolumn{5}{|l|}{ Differentiation } \\
\hline Well & $0(0)$ & $1(33.3)$ & $2(66.7)$ & \multirow{3}{*}{0.637} \\
\hline Moderately & $0(0)$ & $13(30.2)$ & $30(69.8)$ & \\
\hline Poorly & $0(0)$ & $0(0)$ & $4(100)$ & \\
\hline \multicolumn{5}{|l|}{ Invasion depth } \\
\hline $\mathrm{T} 4$ & $0(0)$ & $1(9.1)$ & $10(90.9)$ & \multirow{3}{*}{0.296} \\
\hline T3 & $0(0)$ & $11(34.4)$ & $21(65.6)$ & \\
\hline $\mathrm{T} 2-\mathrm{T} 1$ & $0(0)$ & $2(28.6)$ & $5(71.4)$ & \\
\hline \multicolumn{5}{|l|}{ Vascular invasion } \\
\hline Negative & $0(0)$ & $13(48.1)$ & $14(51.9)$ & \multirow{2}{*}{$0.001 *$} \\
\hline Positive & $0(0)$ & $1(4.3)$ & $22(95.7)$ & \\
\hline \multicolumn{5}{|l|}{ Lymph node metastasis } \\
\hline Negative & $0(0)$ & $7(30.4)$ & $16(69.6)$ & \multirow{2}{*}{0.726} \\
\hline Positive & $0(0)$ & $7(25.9)$ & $19(74.1)$ & \\
\hline \multicolumn{5}{|l|}{ Stage } \\
\hline I-II & $0(0)$ & $7(29.2)$ & $17(70.8)$ & \multirow{2}{*}{0.860} \\
\hline III- IV & $0(0)$ & $7(26.92)$ & $19(73.07)$ & \\
\hline \multicolumn{5}{|l|}{ Stage subgroups } \\
\hline I & $0(0)$ & $2(40)$ & $3(60)$ & \multirow{7}{*}{0.963} \\
\hline III & $0(0)$ & $5(29.4)$ & $12(70.6)$ & \\
\hline IIb & $0(0)$ & $0(0)$ & $2(100)$ & \\
\hline IIIa & $0(0)$ & $0(0)$ & $1(100)$ & \\
\hline IIIb & $0(0)$ & $6(33.3)$ & $12(66.7)$ & \\
\hline IIIc & $0(0)$ & $1(16.7)$ & $5(83.3)$ & \\
\hline IVa & $0(0)$ & $0(0)$ & $1(100)$ & \\
\hline \multicolumn{5}{|l|}{ Localization } \\
\hline Left & $0(0)$ & $10(34.5)$ & $19(65.5)$ & \multirow{2}{*}{0.341} \\
\hline Right & $0(0)$ & $4(19)$ & $17(81)$ & \\
\hline
\end{tabular}




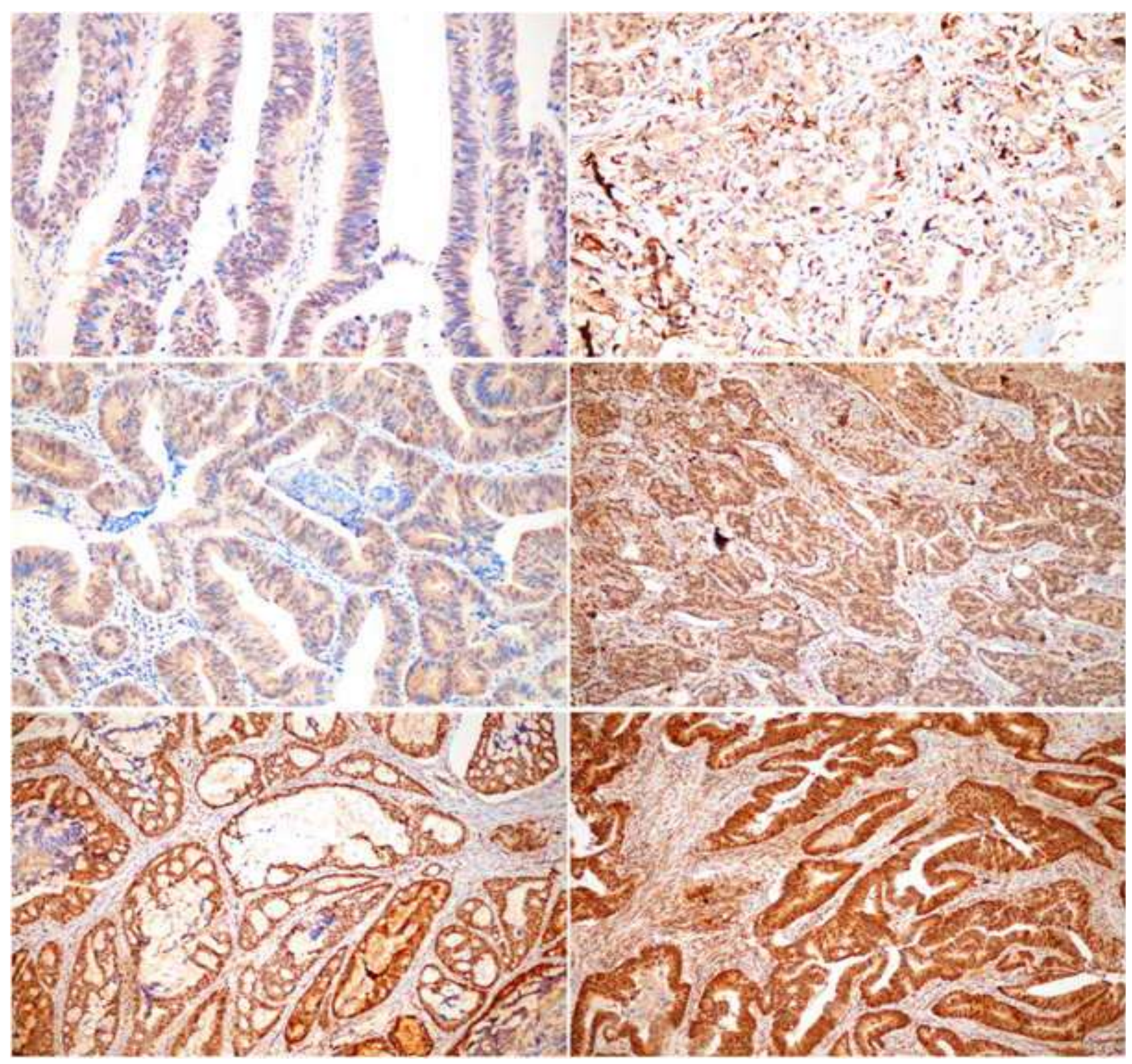

Figure 1. Osteopontin expression; Evaluation for staining intensity; magnifications at x 200 (left to right). a) Score 1 cytoplasmic staining at a villous adenoma. b) Score 1 cytoplasmic staining at a moderately differentiated adenocarcinoma. c) Score 2 cytoplasmic staining at a moderately differentiated adenocarcinoma. d) Score 2 cytoplasmic staining at a moderately differentiated adenocarcinoma. e) Score 3 cytoplasmic staining at an adenocarcinoma. f) Score 3 cytoplasmic staining at a moderately differentiated adenocarcinoma.

\section{Discussion}

Osteopontin is a sialic acid-rich phosphoglycoprotein as well as an extracellular matrix protein that binds integrins at the structure [9]. Since its discovery, extensive research has been conducted on its differing roles, as it is considered to be more than a regulatory protein in normal physiological functions; it has also been shown in various studies to function in apoptosis, proliferation, adhesion, invasion, and tumor metastasis [10,11]. Many studies have shown that osteopontin is highly expressed in various cancers (such as breast, lung, prostate carcinomas, osteosarcoma, glioblastomas, and melanomas), which is useful in different ways 
[6,7]. Similarly, there are many reports of increased osteopontin expression in the regulation of cancer invasion, intraextravasation, and colonization for distant tissues [12,13]. We attempted to obtain meaningful results about the above parameters that would provide useful clues during patient diagnosis and follow-up after surgical resection.

This study found the score 3 staining ratio (72\%) to be significantly higher in the malignant population than in the polyp group (31.2\%). However, score 2 staining was higher in the polyp group $(68.8 \%)$ than in the malignant group (28\%). In tumoral cases, osteopontin staining intensity was significantly higher in the scoring system than with polyp cases $(p=0.001)$. While insignificant, differences were found according to differentiation grade, with scoring 3 staining patterns present in $66 \%$ of well-differentiated tumors rising to universal occurrence among poorly differentiated cases.

Vascular invasion is an established adverse prognostic factor in colorectal and other carcinomas [14]. Wei et al. have identified the top 15 genes (especially OLR1, GPNMB, PRRX1, and BCAT1) that co-upregulate with OPN in human colon cancers pecimens, promoting cancer migration and invasion in various types of cancer [15]. A study carried out with hepatocellular carcinoma specimens suggested that OPN overexpression independently correlates with vascular invasion and predicts poor survival in patients undergoing hepatectomy for hepatocellular carcinoma [16]. In our study, we found that score 3 stainings were higher $(95.7 \%)$ in patients with vascular invasion than those without $(51.9 \% ; p=0.001$, confirming that hypothesis. In a study with 82 colorectal carcinoma patients, Likui et al. found a significant correlation between osteopontin mRNA expression and lymph nodes so that venous metastases [17]. We could not find a significant correlation related to lymph node metastasis, but this may be due to having an insufficient number of patients. We did find that OPN staining was not associated with age or gender ( $p=0.059$ and $p=0.649$, respectively) in tumors, polyps, and normal tissues (Table 2). We also observed significant differences in staining percentage and intensity scores across various parameters of osteopontin expression. However, some of them probably did not reach statistically significant levels, which might also be due to the number of cases investigated.

Although the number of polyps in the study group was low, there was a significant difference in staining compared to patients with tumors. To find out whether an intramucosal carcinoma focus is present or has been developing, the widespread and intense staining of osteopontin will provide a meaningful warning sign when evaluating an adenomatous polyp biopsy.

Vascular invasion is a significant negative prognostic factor for all cancers and colon cancer in particular. Pathologists may be unable to observe vascular invasion because of vascular invasion not being present in examined sections. Therefore, intense staining of the osteopontin can be beneficial when detecting vascular invasion.

\section{Conclusions}

We suggest that osteopontin could be a beneficial biomarker in predicting vascular invasion. If supported by an adequate case series, it may also provide valuable information regarding invasive-precursor distinction. Hence, high staining score with osteopontin could provide useful information about predicting prognosis and determining the treatment method of colon cancer. 
Funding: There is no financial support and sponsorship

Conflict of Interest: The authors declare that they have no conflict of interest.

Ethical statement: The study was approved by Dışkapı Yıldırım Beyazıt Training and Research Hospital local ethics committee (Decision Number 25/08).

\section{Open Access Statement}

This is an open access journal which means that all content is freely available without charge to the user or his/her institution under the terms of the Creative Commons Attribution NonCommercial License

(http://creativecommons.org/licenses/bync/4.0). Users are allowed to read, download, copy, distribute, print, search, or link to the full texts of the articles, without asking prior permission from the publisher or the author.

\section{References}

[1]Hamilton SR, Bosman FT, Boffeta P. Colon and rectum. Bosman FT, Carneiro F, Hruban $\mathrm{RH}$, editors. Tumors of the colon and rectum. WHO Classification of tumours of the Digestive System, 4th ed. Lyon: 2010. p.134.

[2]Senger DR, Wirth DF, Hynes RO. Transformed mammalian cells secrete specific proteins and phosphoproteins. Cell. 1979; 16(4):885-93.

[3]Wai PY, Kuo PC. The role of Osteopontin in tumor metastasis. J Surg Res. 2004; 121(2):228-41.

[4] Weber GF, Lett GS, Haubein NC. Osteopontin, is a marker for cancer aggressiveness and patient survival. $\mathrm{Br} \mathbf{J}$ Cancer. 2010; 103(6):861-69.

[5]Weber GF. The cancer biomarker osteopontin: combination with other markers. Cancer Genomics

Proteomics. 2011; 8(6):263-88.

[6]Wei R, Wong JPC, Kwok HF. Osteopontin, a promising biomarker for cancer therapy. $\mathrm{J}$ Cancer. 2017; 8(12)2173-83.

[7]Shevde LA, Samant RS. Role of osteopontin in the pathophysiology of cancer. Matrix Biol. 2014; 37:131-41.

[8]Allred DC, Bustamante MA, Daniel CO, et al. Immunocytochemical analysis of estrogen receptors in human breast carcinomas. Evaluation of 130 cases and review of the literature regarding concordance with biochemical assay and clinical relevalence. Arch Surg. 1990; 125(1):107-13.

[9]Craig AM, Smith JH, Denhardt DT. Osteopontin, a transformation-associated cell adhesion phosphoprotein, is induced by 12-O-tetradecanoylphorbol 13-acetate in mouse epidermis. J Biol Chem. 1989; 264(16):9682-89.

[10] Mazzali M, Kipari T, Ophascharoensuk V, et al. Osteopontin a molecule for all seasons. QMJ. 2002; 95(1):3-13.

[11]Rittling SR, Chambers AF. Role of osteopontin in tumour progression. $\mathrm{Br} \mathrm{J}$ Cancer. 2004; 90(10):1877-81.

[12] Rangaswami H, Bulbule A, Kundu GC. Osteopontin: role in cell signalling and cancer progression. Trends Cell Biol. 2006; 16(2):79-87.

[13] Hirama M, Takahashi F, Takahashi K, et al. Osteopontin overproduced by tumour cells acts as a potent angiogenic factor contributing to tumour growth. Cancer Lett. 2003; 198(1):107-17.

[14] Jessup JM, Goldberg RM, Asare EA. Colon and rectum. Amin MB, Edge SB, Greene FL, editors. AJCC Cancer Staging Manual Eight Edition. Chicago: Springer. 2017. p.251274. 
[15] Wei R, Wong JPC, Lyu P, et al. In Vitro and Clinical Data Analysis of Osteopontin as a Prognostic Indicator in Colorectal Cancer. J Cell Mol Med. 2018. 22(9):4097-105.

[16] Korita PV, Wakai T, Shirai Y, et al. Overexpression of osteopontin independently correlates with vascular invasion and poor prognosis in patients with hepatocellular carcinoma. Hum Pathol. 2008;39(12):1777-83.

[17]Likui W, Hong W, Shuwen Z. Clinical significance of the upregulated osteopontin mRNA expression in human colorectal cancer. J Gastrointest Surg. 2010;14(1):7481 . 